\title{
Demonstration of dual gain mechanism in an InCaAs/InAlAs superlattice photodiode
}

\author{
W-Q. Li, Paliab K. Bhattacharya, and F-Y. Juang \\ Sold State Electronics Laboratory, Department of Elecrical Engineering and Computer Science, The \\ University of Michigan, Anp Ambor, Michigan 48109-2122
}

(Received 22 December 1986; accepted for publication 27 February 1987)

\begin{abstract}
A high-gain photodiode in which the internal gain can result from either potential barrier lowering or thass fltering action, depending on device geometry and bias conditions, is proposed and demonstrated. The photodiode structure is simiar to modulated barrier diode and uses $\ln _{0.53} \mathrm{Ga}_{0.47} \mathrm{As}$ and InGaAs/InAlAs superlattice absorption regions. The superlattice helps to reduce the dark current and aids in mass filtering. The devices reported here were made with multilayered Inp-based materials grown by molecular beam epitaxy and exhibit responsivity as high as $1000 \mathrm{~A} / \mathrm{W}$.
\end{abstract}

For many applications, it is desirable to be able to detect very low signal levels with moderate or low response speeds. It is also sometmes important in such applications to have a high responsivity over a large voltage range. We present here the results obtained from a heterojunction photodiode, which can, in principle, exhibit large internal gains by two diferent mechanisms, dependirg on the device design, temperature of operation, and applich bias. These mechanisms are enhanced thermionic emission over a potential barrier ${ }^{1,2}$ and efective mass filtering, ${ }^{3}$ which can separately, or in combination, give rise to large gains over a wide voliage range. The device being reported here exnibits a peak responsivity of $140 \mathrm{~A} / \mathrm{W}$ at $300 \mathrm{~K}$ and $\sim 1000 \mathrm{~A} / \mathrm{W}$ at $50 \mathrm{~K}$ in the spectral range of $1.25-1.55 \mu \mathrm{m}$.

The operating principles of our devices are briefiy described, with respect to the band diagrams shown in Fig. 1 . The device is biased such that the InGaAs layer is positive with respect to the superlattice (SL). If the photoexcitation is incident on the InGaAs side, and is mostly absorbed in this ternary layer, the photogenerated holes drift toward the barrier layer and accumulate there, thereby lowering the barrier height. This results in increased thermionic enission of electrons from the SL to the ternary layer and consequent optical gain. On the other hand, if the device is excited from the $S I$ end, electron-hole pairs are nostly generated in this region. Due to the bias polarity and due to increased confnement and scattering of holes in the quantum wells, all of them may not reach the barrier, and hence efficient barrier lowering may not be achieved. This agrees with our experimental observations. If the total bias and he doping and thickness of the individual layers can be so adjusted such that a combination of the potential drop across the $S t$ and the barrier lowering can tilt its bands in the opposite direction, then photoelectrons will drift in the same direction as the electrons injected from the contact regions. The thicknesses of the wells and barriers of the superlattice are properly chosen, such that electrons are transported by miniband conduction while holes are localized in the wells along the direction perpendicular to the layers and their conduction proceeds by phonon assisted tunneling. This is essentially an effective mass filtering effect in the $S L$ which can yield a high photoconductive gain $G=\tau / t_{r}, t=$ hole lifetime, $t_{r}=$ electron (ransit time) over and above modulated barnier gain. Hence the structure we propose and denonstrate can, in principle, achieve high internal gains by either or both mechanisms.

The devices being reported here were grown by molecular beam epitaxy on S-ioped $n^{+}-$mp substrates. The $n^{+}-n-p^{+}-n-n^{+}$structure is ssentially similar to earlier reported modulated barrier ${ }^{4,5}$ or Camel diodes, with some essential differences. The entire structure is grown with inP. based materials, and one side of the diode contains a superlattice region. The $n-$ InGaAs and superhatice regions are undoped, with an effective electron density of $5 \times 10^{15} \mathrm{~cm}^{-3}$. The $p^{+}$barrier layer has a hole concentration of $5 \times 10^{18}$ $\mathrm{em}^{-3}$ and is 80 A thick. The thickness of the undoped regions is kept small $(0.40 .6 \mathrm{~mm})$ to ensure full depletion at thermal equilibrim. The superlatice is characterized by $Z_{Z}=49 \mathrm{~A}$ and $L_{B}=23 \mathrm{~A}$.

Circular mesa diodes were fabricated by atching with $1 \mathrm{H}_{2} \mathrm{SO}_{4}: 1 \mathrm{H}_{2} \mathrm{O}_{2}: 8 \mathrm{H}_{2} \mathrm{O}(1.5 \mu \mathrm{m} / \mathrm{min})$ through photolithographic masks. Au/Ge contact pads were formed by evaporation and alloying and were deineated by standard lift-of techniques. Typical diode areas were $\sim 5 \times 10^{-4} \mathrm{~cm}^{2}$ and the zero-bias capacitance was $\sim 1.0 \mathrm{pF}$. Typical current-voltage characteristics in the dark and under illumination are shown in Fig. 2. Using the thermionic emission model up to a bias of $2 \mathrm{~V}$, a linear plot of in $I \mathrm{vs} F$ is obtained from which a barrier potential of $0.6 \mathrm{eV}$ is calculated. This is comparable to a value of $0.56 \mathrm{eV}$ measured in GaAs/AlGaAs devices. ${ }^{4}$ We also made homojunction devices with $\ln _{0.53} \mathrm{Ga}_{0.47} \mathrm{As}$,

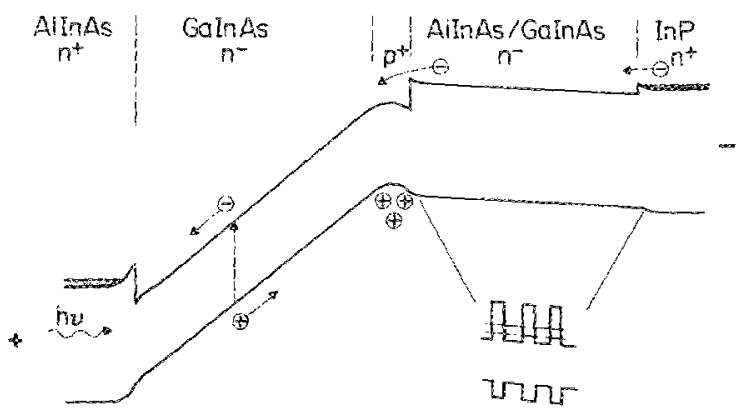

FIG. 1. Band diagrams of an $n-p^{++}-n^{\prime \prime}$ barrier diode under bias. The structures used in this study were grown by molecular beam epitaxy. 


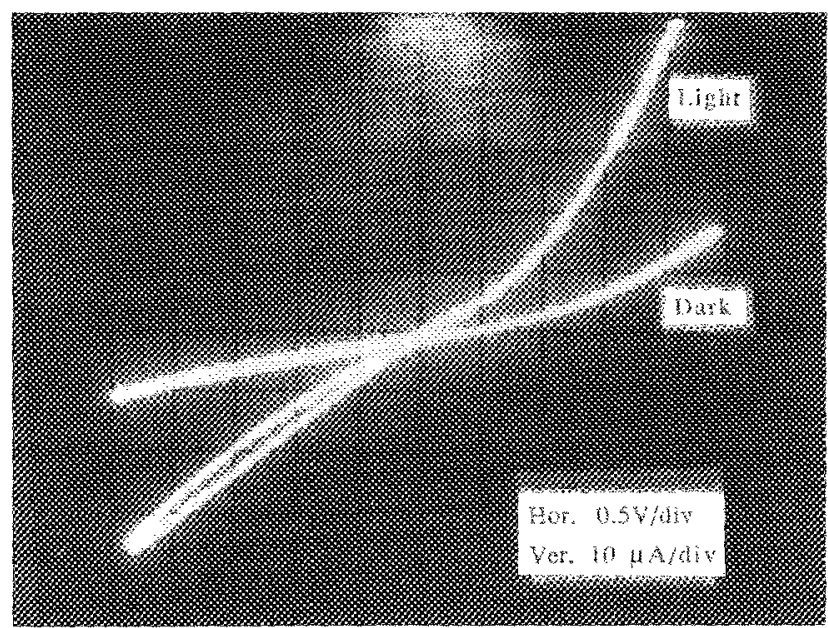

FIG. 2. Current-voltage characteristics in an InGaAs/nAIAs SL modulated carrier photodiode in the dark and under illumination.

but the leakage current in such devices reached untolerable limits. The incorporation of the SL dranaticaliy reduced this leakage, because of the increased band gap and the achievement of material quality enhancement.

The variation of responsivity as a function of bias and temperature in a device (sample A) which has a $0.55 \mu \mathrm{m}$ and $0.4 \mathrm{~mm}$ InGaks and SL regions, respectively, is shown in
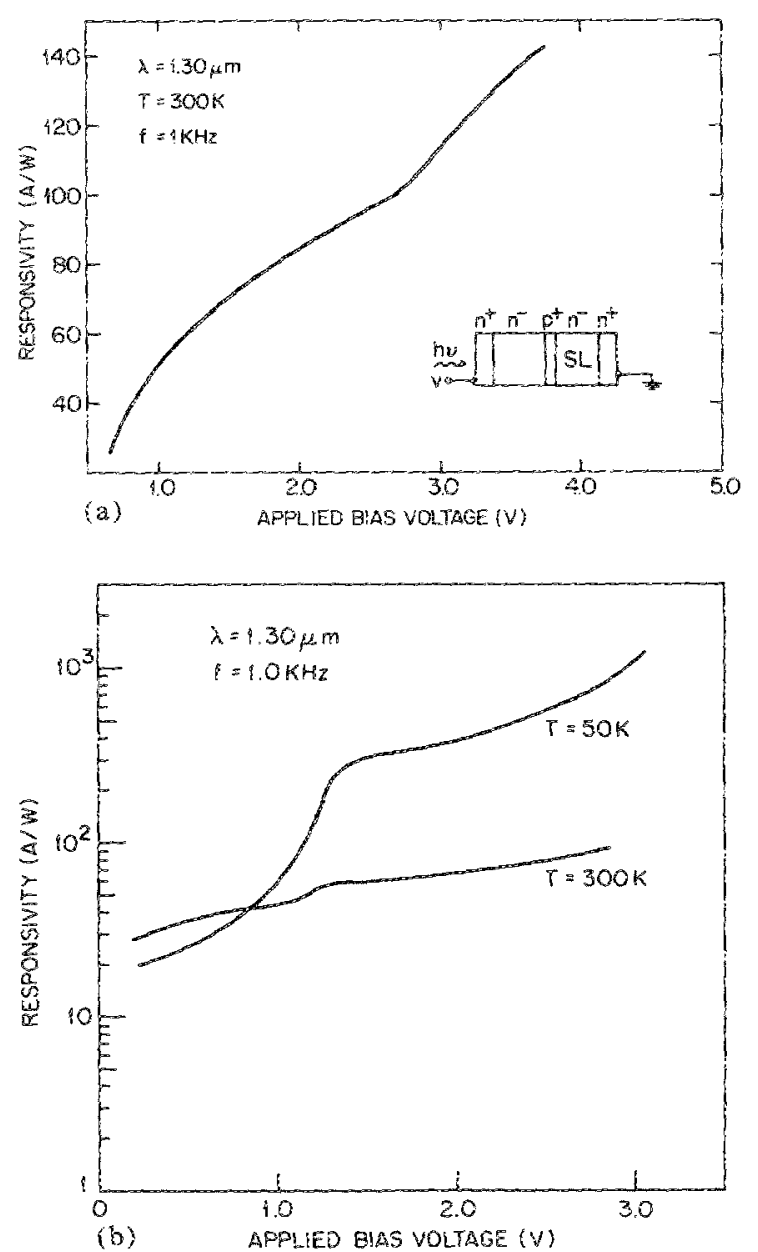

FIG. 3. Variation of responsivity with applied bias (a) at $300 \mathrm{~K}$ and (b) at 300 and $50 \mathrm{~K}$ in different samples.

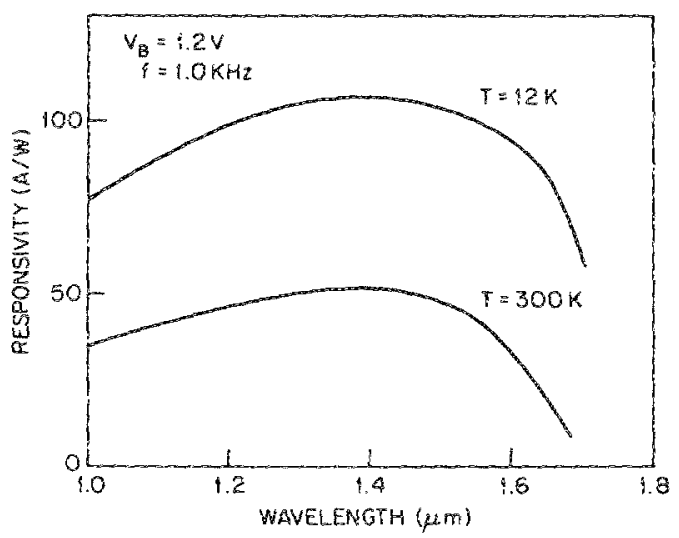

FrG. 4. Spectral response of the photodicde ar 300 and $50 \mathrm{~K}$.

Fig. 3. The $1.3 \mathrm{~km}$ photoexcitation is principally absorbed in the InGas layer and the bias configuration is as shown in Fig. 1 . At room temperature the responsivity increases with bias [Fig. 3 (a)] because of barrier lowering due to a more efficient accumulation of holes in the barrier layer. As the bias is further increased, the band bending in the superiatice region is reduced and this prevents the accumulation of holes in the barrier region. Therefore, a saturation cccurs in the characteristics around $2.5 \mathrm{~V}$. Beyond this bias value there is a second region where the responsivity increases with bias. We believe that efective mass filtering between electrons and holes may be responsible. Figure $3(b)$ depicts the gain at 300 and $50 \mathrm{~K}$ in another sample (b). It is clearly seen that the gain decreases at low biases, which would be true for thermionic emission, and increases at higher biases, confirming mass fittering in this bias range. Furthermore, the measured gains are extremely high and comparable to those reported by Capasso. The kink in the low-temperature responsivity characteristics is not clearly understood and we think this may be a heterostructure effect. It showid also be noted that the data of Figs. $3(a)$ and $3(b)$ were obtained with extremely low light intensities $(\sim \mu W)$. Figure 4 depicts the temperature-dependent spectral response at $I \mathrm{~V}$ bias with illumination from the InGaAs iayer end. The long wavelength cutoff represents the absorption edge of the ternary material and the decrease in responsivity at shorter wavelengths is mainly due to surface absorption effects. The peak response is obtaned in the $1.25-1.55$ krn range and this is representative of absorption in both ternary and superlattice regions.

In conclusion, we have reported the principle and operation of a high-gain photodetector in which a high responsivity can result from two independent internal gain mechanisms which complement each other. We have used an InGaAs/InAlAs SL in one region of an InGaAs modulated barrier photodiode. The $\$ \mathrm{~L}$ not only helps to reduce the reverse leakage current, but with the proper band tilting, can give high photoconductive gains by a mass filtering action.

The authors acknowledge stimulating discussions with Professor H. Beneking and Dr.F. Capasso. The work is being supported by the Defense Adwanced Research Projects Agency and the Amy Research Onice under contract DAAL03-86-K0105. 
J. M. Shannon, Appl. Phys. Lett. 35, 64 (1979).

2. 3. MoCleer and G. I. Haddad, IEEE Trans. Electron Devices ED-25, $389(1978)$

${ }^{3}$ F. Capasso, K. Mohammed, A. Y. Cho, R. Hull, and A. L. Hutchinson, Appl. Plyss. Lett. 47, 420 (1985).

'C. Y. Chen, A. Y. Cho, P. A. Garbinski, C. G. Bethea, and B. F. Levine,
App. Phys. Lett. 39, 340 (1981).

5J. A. Barnard, F. E. Najjar, and L. F. Eastman, IEEE Trans. Electron Devices ED-29, 1396 (1982).

(N. Georgoulas, IEEE Electron Device Lett. EDL-3, 61 (1982).

W. T. Masselink, Y. L. Sun, R. Fischer, T. J. Drummond, Y. C. Chang, M V. Klein, and H. Morkọ, J. Vac. Sci. Technol. B 52, 117 (1984). 